\title{
Ultrasound-sensitizing nanoparticle complex for overcoming the blood-brain barrier: an effective drug delivery system
}

This article was published in the following Dove Press journal: International Journal of Nanomedicine

\author{
Shin-Woo $\mathrm{Ha}^{1,2, *}$ \\ Kihwan Hwang ${ }^{3, *}$ \\ Jun Jin ${ }^{3}$ \\ Ae-Sin Cho' \\ Tae Yoon Kim ${ }^{1,2}$ \\ Sung II Hwang' \\ Hak Jong Lee ${ }^{1,2,4,5}$ \\ Chae-Yong Kim ${ }^{3,6}$
}

'Department of Radiology, Seoul National University College of Medicine, Seoul National University Bundang Hospital, Seongnam I3605, South Korea; ${ }^{2}$ Research Institute, IMGT Co., Ltd, Seongnam, 13605, South Korea;

${ }^{3}$ Department of Neurosurgery, Seoul National University Bundang Hospital, Seongnam-si, South Korea; ${ }^{4}$ Department of Nanoconvergence, Seoul National University Graduate School of Convergence Science and Technology, Seoul, South Korea; ${ }^{5}$ Research Strategy and Management Division and Medical Device R\&D Center, Seoul National University Bundang Hospital, Seongnam 13605, South Korea; ${ }^{6}$ Department of Neurosurgery, Seoul National University College of Medicine, Seoul National University, Seoul, South Korea

*These authors contributed equally to this work

Correspondence: Chae-Yong Kim

Department of Neurosurgery, Seoul National

University College of Medicine, Seoul Nationa

University Bundang Hospital, 82, Gumi-ro 173

Beon-gil, Bundang-gu, Seongnam-si, Gyeonggi-

do 13620 , South Korea

Tel +82317877165

Fax +82 31 7874097

Email chaeyong@snu.ac.kr

Hak Jong Lee

Department of Radiology, Seoul National University College of Medicine, Seoul National University Bundang Hospital, 82, Gumi-ro 173 Beon-gil, Bundang-gu, Seongnam-si, Gyeonggido 13620 , South Korea

Tel +82 31 7877605

Fax +82 31 7878709

Email hakjlee@snu.ac.kr
Background: Crossing the blood-brain barrier (BBB) is crucial for drug delivery to the brain and for treatment of brain tumors, such as glioblastoma, the most common of all primary malignant brain tumors. Microbubble (MB) is oscillated and destroyed by controlling ultrasound (US) parameters. This oscillation and destruction of MB can open the BBB transiently, and a drug can be delivered to the brain.

Materials and methods: For testing the efficiency of delivery to the brain, we synthesized a US-sensitizing nanoparticle (NP) complex via chemically binding MBs and NPs for the BBB opening, including near-infrared dye-incorporated albumin nanoparticles (NIR-Alb NPs) for fluorescence detection.

Results: The human-derived, biocompatible NIR-Alb NPs did not show significant cytotoxicity to $500 \mu \mathrm{g} / \mathrm{mL}$ for 3 days in four human glioma cell lines. In an in vivo animal study, some US parameters were investigated to determine optimal conditions. The optimized US conditions were applied in a U87MG orthotopic mouse model. We found that the fluorescence intensity in the brain was 1.5 times higher than in the control group.

Conclusion: Our US-sensitizing NP complex and US technique could become one of the critical technologies for drug delivery to the brain.

Keywords: blood-brain barrier, ultrasound, nanoparticle, microbubble, complex, drug delivery

\section{Introduction}

Glioblastoma (GBM) is the most common of all primary malignant brain tumors in adults. ${ }^{1}$ GBM has a very dismal prognosis with an overall survival of only 14-15 months, even after aggressive multi-disciplinary treatments, including surgery, radiation, and chemotherapy. Many active chemotherapeutics have been developed; however, the utilization of these agents for the treatment of GBM is limited due to the difficulty of delivering these large molecules of over 400-500 Da across the blood-brain barrier (BBB). ${ }^{2}$

Nanoparticles (NPs) have been studied and clinically used as drug carriers because of the remarkable reduction of adverse effects, such as nausea and hair loss. ${ }^{3}$ A passive targeting carrier, such as Doxil, ${ }^{4}$ is delivered to leaky vessels in a solid tumor. Even though nanocarriers allow sustained drug release and long circulation time, crossing the BBB remains a challenge for drug delivery to the brain. ${ }^{5}$ For breaking through this hurdle, chemical, biological, and physical approaches for crossing the BBB have been investigated and studied. ${ }^{6}$ One of the approaches in NP use is a surface modification 


\section{A Complexation of nanoparticle and microbubble}

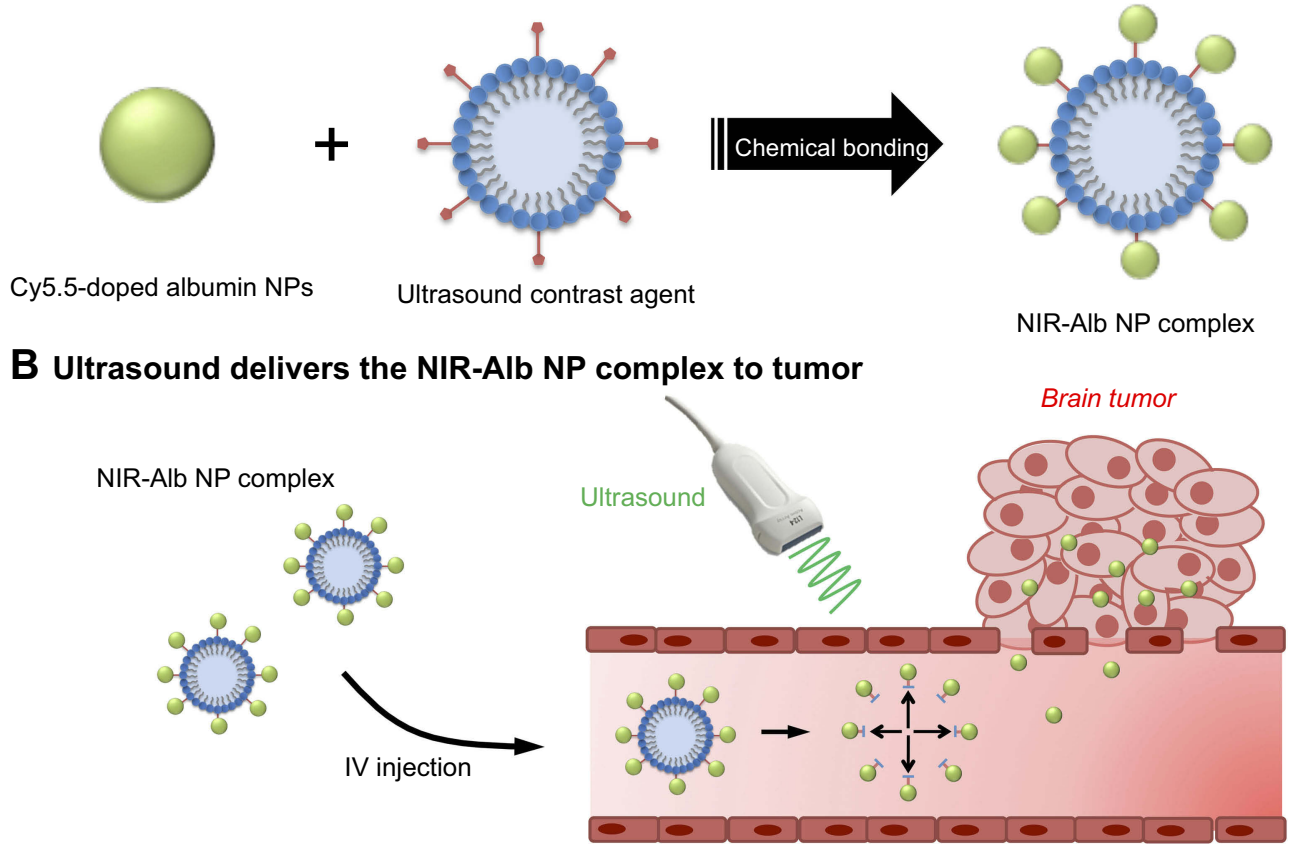

Scheme I Complexation of microbubble and NIR-Alb NP. (A) and NIR-Alb NP complex delivery to brain tumor by ultrasound; (B) NIR-Alb NP can be bound on NHSactivated MB by amide bonding coupling. The as-prepared complex is administrated via tail-vein intravenous injection. When ultrasound is applied in brain tumor site, the complex is oscillated and destroyed, opening the BBB. Finally, the nanoparticle can be delivered to tumor site in the brain.

Abbreviations: BBB, blood-brain barrier; MB, microbubble; NHS, hydroxysuccinimide; NIR-Alb NP, near-infrared dye-incorporated albumin nanoparticle.

with specifically or selectively bound ligands on brain tissues or cells. Transferrin, insulin, and albumin can be used for receptor-mediated and adsorptive transcytosis. Albumin, a biocompatible, endogenous material synthesized in the body, comprising approximately $60 \%$ of all the plasma proteins, is especially useful. ${ }^{7}$ Overexpression and promotion of albumin binding protein, secreted protein acidic and rich in cysteine in GMB was reported. ${ }^{8,9}$

Among the physical methods for the BBB opening, ultrasound (US) is a clinically feasible method because it can presently be used as a diagnostic tool and therapeutic modality. ${ }^{6,10}$ BBB opening and various disease treatments by US, including focused ultrasound (FUS), have been studied, ${ }^{11-16}$ controlling key parameters of FUS, such as frequency ${ }^{17}$ and acoustic pressure. ${ }^{18}$ Since the combination of microbubbles (MBs) and FUS with lower energy for reducing tissue damage by thermally induced BBB were first demonstrated, ${ }^{19}$ optimization conditions of US for safe BBB opening has been investigated, including types of $\mathrm{MBs},{ }^{16}$ size and numbers of $\mathrm{MBs},{ }^{20}$ and various US parameters. ${ }^{21-23}$ Furthermore, several studies demonstrated that a diagnostic US system could open the BBB with MBs. ${ }^{23-25}$

To overcome the limitation of drug delivery across the BBB, we designed US-sensitizing NP complexes, which were created by complexing near-infrared (NIR) dye-doped albumin NPs with N-hydroxysuccinimide (NHS)functionalized MBs (Scheme 1A). Albumin NPs were synthesized by the desolvation method. For diminishing autofluorescence in tissue, NIR dyes were introduced into the NPs. In addition, for efficient delivery using our USsensitizing NP complex (near-infrared dye-incorporated albumin nanoparticle [NIR-Alb NP] complex), US parameters for a normal mouse brain were tested and optimized. Finally, we applied this complex to a U87 MG orthotopic mouse model and confirmed the efficient nanoparticle delivery under the optimized US parameters through fluorescence detection using an in vivo imaging system (IVIS).

\section{Materials and methods}

\section{Synthesis of NIR dye-doped albumin NPs (NIR-Alb NPs)}

Synthesis of Cy5.5-doped albumin NPs was slightly modified from Langer's methods. ${ }^{26} 1 \mathrm{~g}$ of human serum albumin (HSA, MO, Sigma-Aldrich) was dissolved in $34 \mathrm{~mL}$ of deionized water. $76 \mu \mathrm{L}$ of Cy5.5-NHS ester (Lumiprobe, MD, USA, $34.9 \mathrm{mM}$ in DMSO) was added. $100 \mathrm{~mL}$ of ethanol anhydrous (Daejung, Korea) was added 
for 5 mins. After $1 \mathrm{hr}, 400 \mu \mathrm{L}$ of $8 \%$ glutaraldehyde (Sigma-Aldrich, MO, USA) was added. $16 \mathrm{hrs} \mathrm{later,} \mathrm{the}$ solution was centrifuged at $15,000 \mathrm{rpm}$ for $15 \mathrm{mins}$. Supernatant was discarded, and the precipitates were dispersed in deionized water. Centrifugation and redispersion were repeated twice. Finally, the dispersed solution was centrifuged at 3,000 rpm for 5 mins to remove aggregates. Supernatant, NP solution was taken. The prepared NPs were characterized by dynamic light scattering (DLS), zeta potential (Malvern Zetasizer NanoZS90, UK), and bio-TEM (TECNAI G ${ }^{2}$ spirit Twin, FEI, USA).

\section{Quantitative analysis of NIR-Alb NP}

Dye concentration was quantified by UV-Visible spectrometer (Shimadzu UV-1800, Kyoto, Japan). Sulfo-Cy 5.5-NHS ester (Lumiprobe, MD, USA) was dissolved in deionized water. Cy5.5 shows a characteristic absorption spectrum in visible range, in which the maximal wavelength is at $694 \mathrm{~nm}$. Using this wavelength, various concentrated solution for the standard curve was measured in deionized water, ranging from 0.61 to $19.5 \mu \mathrm{g} / \mathrm{mL}$ with 6 points.

\section{Synthesis of MBs}

MBs were synthesized by phospholipid thin film hydration method. ${ }^{27}$ 1,2-distearoyl-sn-glycero-3-phosphocholine (DSPC, NOF, Japan) and 1,2-distearoyl-sn-glycero3-phosphoethanolamine-N-[succinyl(polyethylene glycol)2000] (DSPE-PEG2k-NHS, NOF, Japan) were dissolved in chloroform at a 9:1 molar ratio. And chloroform was evaporated to form thin phospholipid thin film. This thin phospholipid film at the concentration of $0.5 \mathrm{mg} / \mathrm{mL}$ was hydrated using $0.9 \% \mathrm{NaCl}$ solution (saline, JW Pharmaceutical, Korea) over phase transition temperature of DSPC $\left(55^{\circ} \mathrm{C}\right)$ by bath sonicator. And the headspace of the vial was filled with sulfur hexafluoride gas $\left(\mathrm{SF}_{6}\right.$, Dong-A Specialty Gases \& Dong-A Scientific, Korea). Sequentially, hydrated liposomal precursor was agitated by Vialmix ${ }^{\mathrm{TM}}$ (Lantheus Medical Imaging, MA, USA) for $45 \mathrm{~s}$, in order to transform to MB. The MBs were characterized by DLS (Malvern Zetasizer NanoZS90, UK), optical microscope (BX-43, Olympus, Japan), and bio-TEM (TECNAI G ${ }^{2}$ spirit Twin, FEI, USA).

\section{Complexation of NP and MB (NIR-Alb NP complex)}

$200 \mu \mathrm{L}$ of well-dispersed NIR-Alb NP solution was added into the as-prepared MB solution with a pressure equalization. 2 hrs after gentle rotation using tube revolver
(ThermoFisher Scientific, MA, USA), the NIR-Alb NP complex was formed. The NIR-Alb NP complexes were characterized by DLS (Malvern Zetasizer NanoZS90, UK) and bio-TEM (TECNAI G ${ }^{2}$ spirit Twin, FEI, USA).

\section{Negative staining of MB and NIR-Alb NP complex for TEM analysis}

MB or NIR-Alb NP complex was applied to glow-discharged carbon-coated copper grids (200 mesh, Ted Pella, USA). After allowing the sample to absorb for 2 mins and blotting off buffer solution onto Whatman paper, then the sample on the grids was stained with $2 \%(\mathrm{w} / \mathrm{v})$ of uranyl acetate (UrAc) for $1 \mathrm{~min}$. Then, it was blotted off UrAc. These results were recorded with the Tecnai G2 Spirit Twin microscope (FEI, USA) at an acceleration voltage of $120 \mathrm{kV}$.

\section{Cell culture, cell viability test of the NPs} Human glioma cell lines U87MG, U251MG, U373MG, and LN-229 were originally purchased from American Type Culture Collection (ATCC, Manassas, VA, USA). These cell lines were cultured in complete DMEM (Sigma-Aldrich, Germany) that was supplemented with 10\% FBS. All cells were incubated at $37^{\circ} \mathrm{C}$ in a humidified atmosphere of $5 \% \mathrm{CO}_{2}$.

The cell viability and cell apoptosis assays were performed with U87 MG, U251 MG, U373 MG, and LN-229 cell lines. The cytotoxicity of NIR-Alb NP was measured using the Cell Counting Kit-8 (CCK-8; Dojindo Molecular Technologies Inc., Tokyo, Japan). CCK-8 contains Dojindo's highly water-soluble tetrazolium salt (WST-8), which produces a water-soluble formazan dye upon reduction in the presence of an electron mediator. Each cell line was seeded into 96-well plates at a density of $5 \times 10^{3}$ cells/ well to allow for overnight adhesion. Subsequent to adhesion, the cells were treated with NIR-Alb NPs at a concentration of $10,30,50,100$, and $500 \mu \mathrm{g} / \mathrm{mL}$ at $37^{\circ} \mathrm{C}$ and $5 \% \mathrm{CO}_{2}$. After 2 days, $10 \mu \mathrm{L}$ of the CCK-8 solution was added to each well of the plate and then the plate was incubated for $2 \mathrm{hrs}$ in an incubator $\left(37^{\circ} \mathrm{C} ; 5 \%\right.$ $\mathrm{CO}_{2}$ ). The OD of the sample plate was measured at $450 \mathrm{~nm}$ by an ELISA reader (VERSAmax microplate reader, Molecular Device, CA, USA). The viability of the tumor cells was assessed by calculating the OD ratio of the specific OD in each sample to the OD of the control sample.

\section{Transfection and luciferase assay}

Human glioma cell lines were transfected with pGL4.50 vector (Promega, Madison, WI, USA) containing the firefly 
luciferase gene (luc2) and the antibiotic resistance gene (Hygro) by using Fugene HD (Promega, Madison, WI, USA). The day before transfection, U87MG cell was plated at a density of $1-2 \times 10^{6}$ cells in $100-\mathrm{mm}$ diameter culture plates. Transient transfection of pGL4.50-luc2 vector was performed on cells growth to $50-80 \%$ confluency using Fugene HD. Luciferase-transfected cells were cultured in the presence of hygromycin B $(400 \mu \mathrm{g} / \mathrm{mL}$; Life Technologies, Waltham MA, USA). After 48 hrs of transfection, the $1 \times 10^{6}$ cells were lysed in 1x passive lysis buffer (Promega, Madison, WI, USA) and luciferase activity was measured using the luciferase assay system (Promega, Madison, WI, USA) and a luminometer reader (Microplate reader luminescence, Molecular Device, CA, USA) according to the manufacturer's instructions.

\section{Orthotopic animal model study}

All experiments were approved by the Institutional Animal Care and Use Committee in Seoul National University Bundang Hospital (SNUBH-IACUC) and animal care and use are conducted by the regulation and the notification of SNUBH-IACUC based on "SNUBH Preclinical Research Center Standard Operating Procedure". Those regulations, notifications, and guidelines are originated and modified from the Guide for the Care and Use of Laboratory Animals 8th edition, NRC (2010).

The authors used U87-luc cell line to make the orthotopic glioma mouse model. Six-week-old female BALB/ c-nu mice were ordered from Orient Bio (Seongnam-si, Korea; distributor for Charles River, Wilmington, MA) and maintained at Seoul National University Bundang Hospital Preclinical Research Center in temperatures of $18-22^{\circ} \mathrm{C}$ and an atmosphere of supply air filtered using a HEPA Filter Unit. A total of 10-15 fresh air changes per hour were provided in the animal housing rooms with the $100 \%$ Outdoor Air System in a positive pressure room.

First, mice were anesthetized with Zoletil 50 (Virbac, Carros, France). Then, the head was fixed in a stereotactic frame, followed by midline scalp incision $(1 \mathrm{~cm})$ to expose the skull. A small hole was made into the skull $0.5 \mathrm{~mm}$ anterior and $2 \mathrm{~mm}$ lateral from the exposed bregma. A sterile $10-\mu \mathrm{L}$ Hamilton syringe with a \#26S needle was inserted at a depth of $3.5 \mathrm{~mm}$ from the surface of the skull and withdrawn by $0.5 \mathrm{~mm}$ to inject $2 \times 10^{5}$ U87MG-luc cells in a volume of $2 \mu \mathrm{L}$. The injection rate was set at $0.5 \mu \mathrm{L} /$ min. After the implantation of the tumor cells, the needle was kept in place for 5 mins to prevent cell reflux. Then, the needle was completely withdrawn from the brain over the course of $3 \mathrm{mins}(1.0 \mathrm{~mm} / \mathrm{min})$. The hole in the skull was sealed with Poly-F Zinc Polycarboxyl Cement (Dentsply Sirona, PA, USA) and the skin was sutured. All of these steps were followed in our lab protocol. This study was approved by the Institutional Animal Care and Use Committee of the Medical Science Research Institute, Seoul National University Bundang Hospital (authorization number: BA1411-164/058-01).

\section{In vivo monitoring}

In vivo image was performed with the IVIS Lumina II coupled Living Image software for data acquisition (Caliper Life Sciences, MA USA). Mice were anesthetized with Zoletil 50 and imaged 12 mins after intraperitoneal injection of luciferin substrate (Promega, Madison, WI, USA). Signal intensity was quantified within a region of interest over the head that was defined by the Living Image software.

\section{Results \\ Synthesis and characterization of NIR-Alb NPs}

Each NIR-Alb NP and MB was synthesized for our USsensitizing NP complexes, and then both were complexed (Scheme 1A). First, NIR-Alb NPs were synthesized by desolvation method. Cy5.5 NHS ester was mixed with albumin solution. Amide bonding was created by a coupling reaction between activated carboxylate groups in the dye and amine groups in albumin. After forming nano-sized albumin aggregates by ethanol, glutaraldehyde was added as a cross-linker between albumins. The NIRAlb NP was $191.4 \pm 79.6 \mathrm{~nm}$ in size and $-49.0 \pm 7.5 \mathrm{mV}$ in zeta potential. The average yield was $56.7 \%$ after the final purification.

To quantify the concentration of Cy5.5 dye in albumin NPs, we created a standard curve using Sulfo-Cy5.5-NHS dyes in water. The standard curve was created by various concentrations, and the maximal absorbance was at $694 \mathrm{~nm}$, the wavelength known as a characteristic peak of the Cy5.5 dye. The average incorporation efficiency was $21.9 \%$. Even though the dye was incorporated, its shape of absorption in the UV-Visible spectrum was not changed (Figure S1).

\section{Synthesis of MBs and complexation of NIR-Alb NPs and MBs}

To form amide bonding between MB and NIR-Alb NPs, NHS-functionalized MB (NHS-MB) was synthesized. First, 

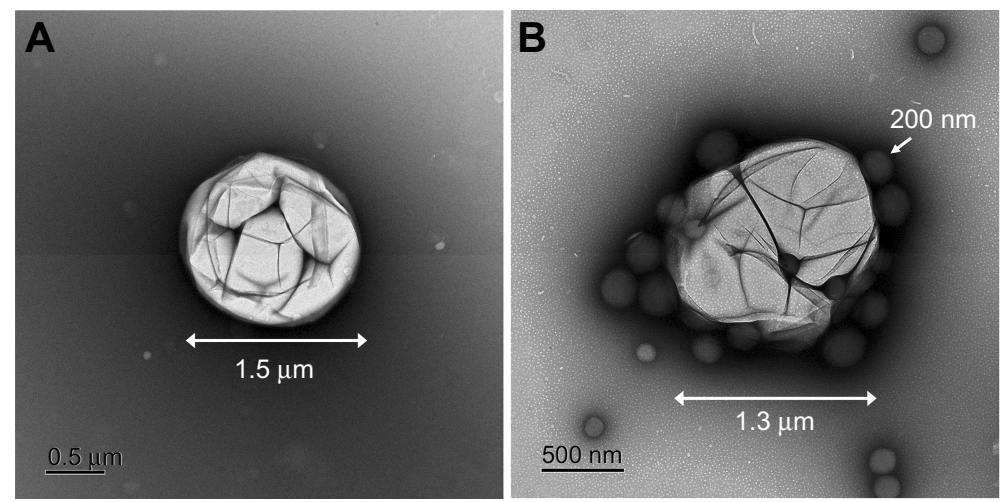

Figure I TEM images of microbubble (A) and US-sensitizing nanoparticle complex (NIR-Alb NP complex) (B). TEM images were taken in negative staining. Abbreviation: NIR-Alb NP, near-infrared dye-incorporated albumin nanoparticle; TEM, transmission electron microscopy.

a lipid film with DSPC and DSPE-PEG-NHS (9:1 molar ratio) was formed. The lipid film was transformed to NHSMBs using the Vialmix. The NHS-MBs were $1.4 \pm 0.3 \mu \mathrm{m}$ by DLS (Figure S2), and their morphology was characterized by TEM (Figure 1A). For the complexation, NIR-Alb NPs were added to the as-prepared NHS-MB solution and gently mixed by hand for 2 hrs. The size of the complex was 2.1 $\pm 0.6 \mu \mathrm{m}$ by DLS (Figure S2), and its morphology was characterized by TEM (Figure 1B). It also was confirmed that the NPs were well decorated on the MB.

\section{Cell viability test of NIR-Alb NPs in human glioma cell lines}

We evaluated the cytotoxicity of NIR-Alb NPs on a human glioma cell line before the in vivo animal study. The genetic biomarkers of GBM cell lines considered were mutation in TP53, phosphatase and tensin homolog (PTEN) deletions, $O^{6}$-methylguanine DNA methyltransferase $(M G M T)$ promoter methylation status and isocitrate dehydrogenase 1 and 2 (IDH1 and IDH2) mutations, epidermal growth factor receptor $(E G F R)$ amplification, $1 \mathrm{p} /$ $19 \mathrm{q}$ deletions, and Ki-67 labeling index. We selected some cell lines that had different biomarker properties and could also form tumors in nude mice. NIR-Alb NPs were treated for 24,48 , and $72 \mathrm{hrs}$ at a concentration of 10, 30, 50, 100, and $500 \mu \mathrm{g} / \mathrm{mL}$ in GBM U87MG (p53 wildtype, $M G M T$ promoter methylation, PTEN mutation) U251MG (p53 mutation, $M G M T$ promoter methylation, PTEN mutation), U373MG (p53 mutation, $M G M T$ promoter methylation, PTEN mutation), and LN229 (p53 mutation, MGMT promoter methylation, $P T E N$ wildtype) cell lines. In Figure 2, NIR-Alb NPs showed over $80 \%$ of cell viability in all of the cell lines, even at the highest dose, that is, $500 \mu \mathrm{g} / \mathrm{mL}$ for 3 days.

\section{Optimization of US parameters for crossing BBB}

To optimize US parameters for crossing the BBB without any damages by US and for effectively delivering NPs, adjustable US parameters, including intensity, treated time, and duty cycle, were tested in normal nude mice. An unfocused US device was used (Sonidel SP100; $1 \mathrm{MHz}$ frequency, intensity $0.1-5 \mathrm{~W} / \mathrm{cm}^{2}$, pulse frequency $100 \mathrm{~Hz}$, duty cycle 5-100\%). Delivery efficiency was measured by fluorescence from NIRAlb NPs after sacrificing and isolating brains. Intensity varied from 0.1 to $4.0 \mathrm{~W} / \mathrm{cm}^{2}$. In Figure 3, the highest fluorescence was shown at the lowest intensity of $0.1 \mathrm{~W} / \mathrm{cm}^{2}$. Even though our US instruments could apply an intensity up to $5.0 \mathrm{~W} / \mathrm{cm}^{2}$, all of the mice died during treatment with $5.0 \mathrm{~W} / \mathrm{cm}^{2}$. In Figure S3, serious hemorrhage in brain parenchyma was observed. In treatment times, 1-min treatment with $0.1 \mathrm{~W} /$ $\mathrm{cm}^{2}$ and $50 \%$ duty cycle was the best condition (Figure 4 ). However, the treatment times did not show remarkable differences or any statistical significance. Another parameter was duty cycle from $10 \%$ to $100 \%$. Figure 5 shows that the change of duty cycle was not significantly effective in NP delivery. When both intensity and duty cycle were changed, US with high intensity and 100\% duty cycle caused damages, such as skin burning at the contact site of the US probe and brain swelling.

\section{Brain delivery of NIR-Alb NPs in U87 MG orthotopic mouse model}

To evaluate brain delivery of NIR-Alb NPs, a U87MG orthotopic xenograft mouse model was created using transfected U87MG-luc cells. The GBM U87MG cells were transfected with pGL4.50 vector transiently. The experiment included a group of cells that were cultured without pGL4.50 vector as a negative control group. As shown in Figure S4, U87MG- 

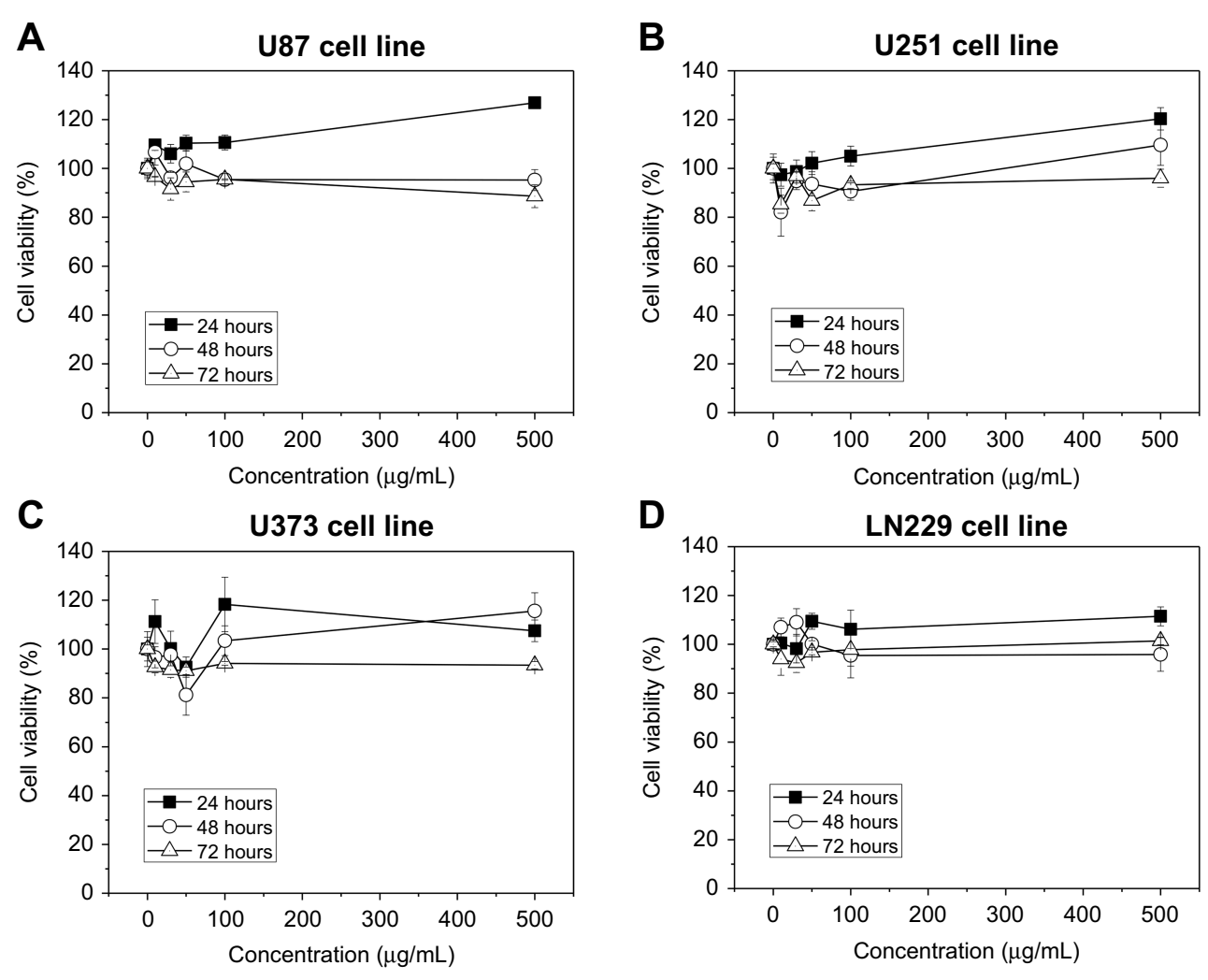

Figure 2 Cell viability of NIR-Alb NPs in U87 (A), U25I (B), U373 (C), and LN229 (D) cell lines. The NPs were treated at a concentration of 10, 30, 50, 100, and 500 $\mu$ g/ $\mathrm{mL}$ lines for 24, 48, and $72 \mathrm{hrs}$.

Abbreviation: NIR-Alb NPs, near-infrared dye-incorporated albumin nanoparticles.
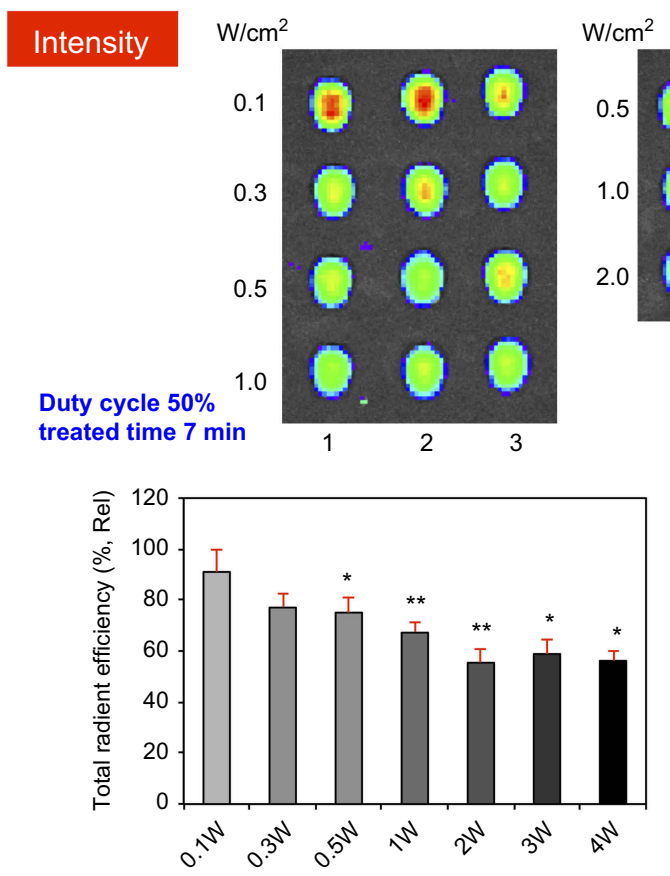

$\mathrm{W} / \mathrm{cm}^{2}$

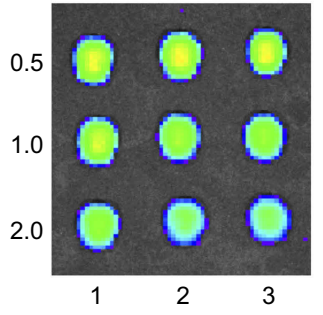

$\mathrm{W} / \mathrm{cm}^{2}$
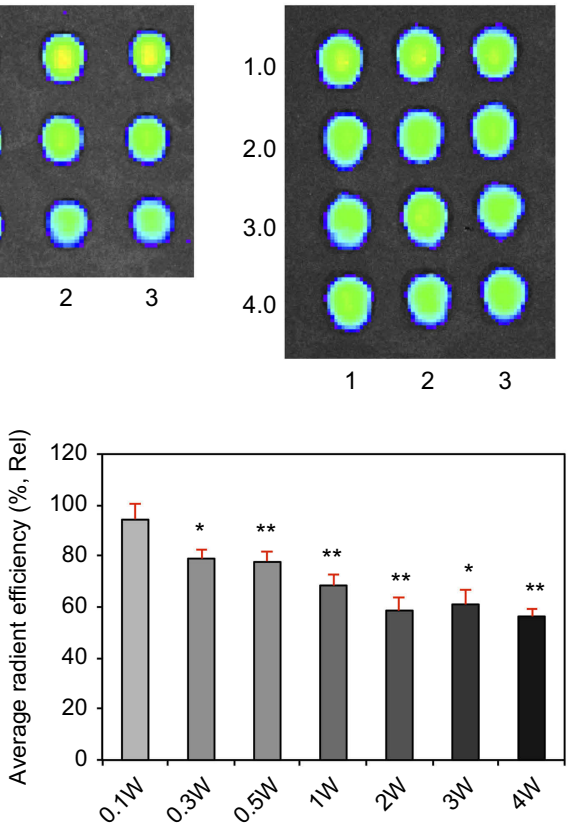

Figure 3 Investigation of various intensities among US parameters from 0.1 to $4 \mathrm{~W} / \mathrm{cm}^{2}$ at $50 \%$ of duty cycle for 7 mins. Fluorescence from Cy5.5 was detected and normalized by IVIS. Comparisons of the intensity were against the $0.1 \mathrm{~W} / \mathrm{cm}^{2}$ using Student's $t$-test $(\mathrm{n}=3)$. $* P<0.05$ and $* * P<0.005$.

Abbreviations: IVIS, in vivo imaging system; US, ultrasound. 


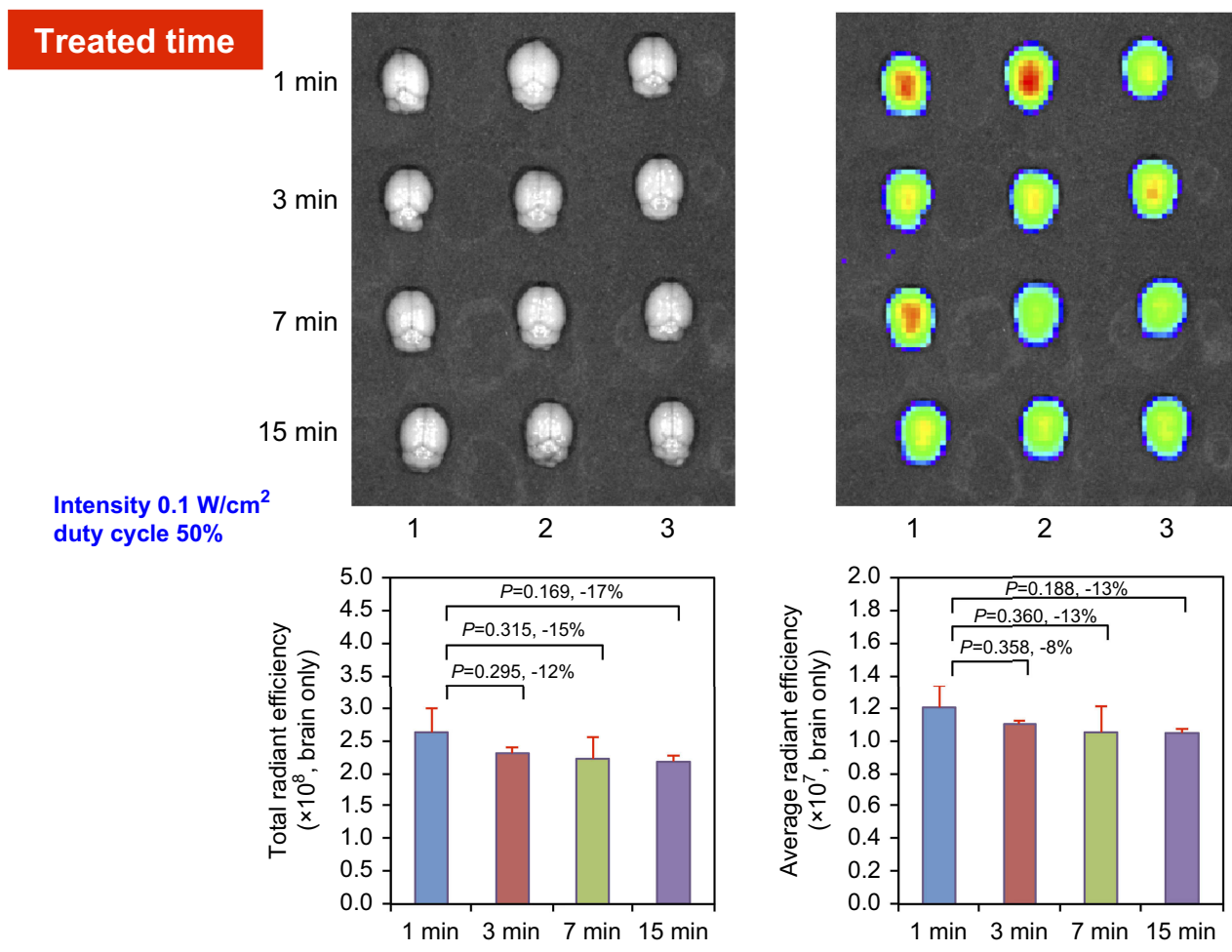

Figure 4 Investigation of various treatment time from I to 15 mins at $50 \%$ of duty cycle and $0.1 \mathrm{~W} / \mathrm{cm}^{2}$ of intensity. Fluorescence from Cy5.5 was detected and normalized by IVIS. Comparisons of the treated time were against the I mins using Student's $t$-test $(n=3) . * P<0.05$ and $* * P<0.005$.

Abbreviation: IVIS, in vivo imaging system.
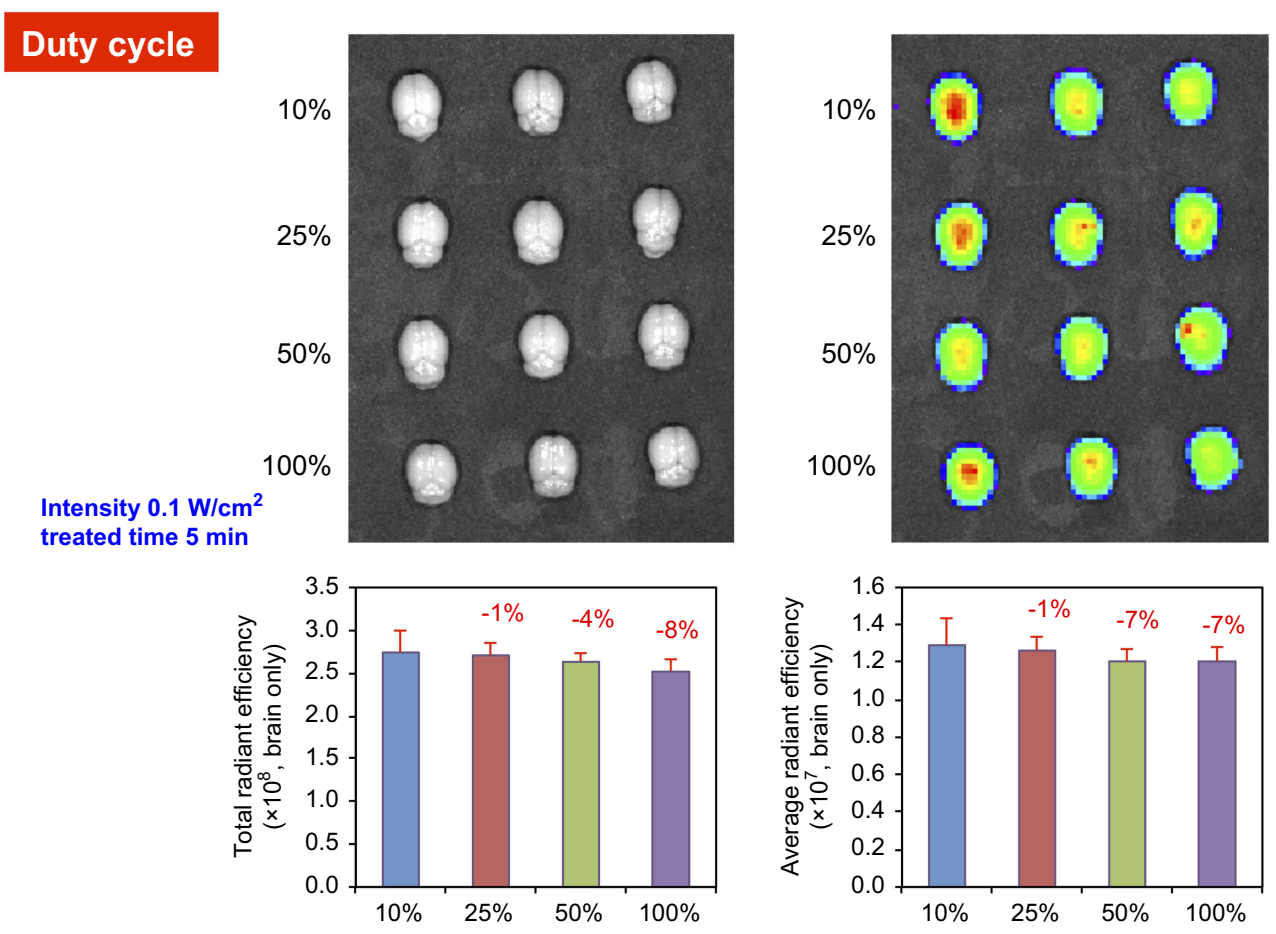

Figure 5 Investigation of various duty cycle from $10 \%$ to $100 \%$ at $0.1 \mathrm{~W} / \mathrm{cm}^{2}$ of intensity for 5 mins. Fluorescence from Cy5.5 was detected and normalized by IVIS. Comparisons of the duty cycle were against the $10 \%$ using Student's $t$-test $(n=3)$. $* P<0.05$ and $* * P<0.005$.

Abbreviation: IVIS, in vivo imaging system. 


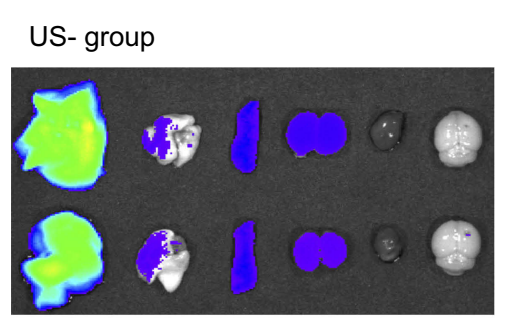

Brain only
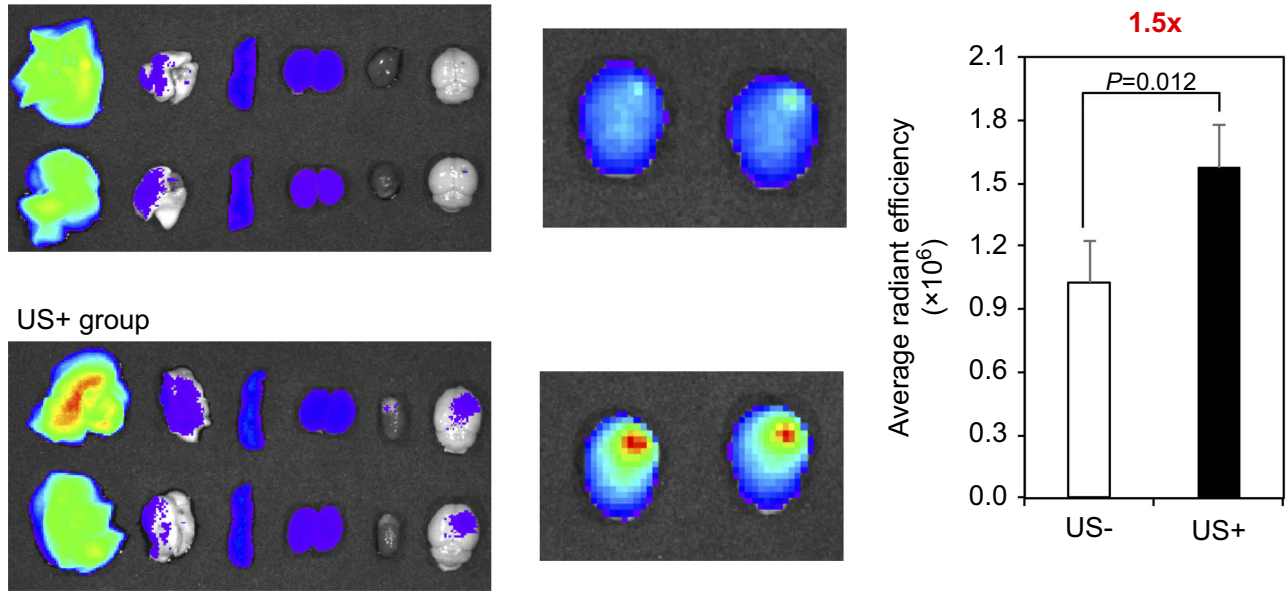

Figure 6 Evaluation of delivery efficiency using NIR-Alb NP complexes in U87 MG-Luc orthotopic mouse model. US was applied at 0.1 W/cm ${ }^{2}$ and $10 \%$ duty cycle for 5 -min treatment on the right side of the brain. Fluorescence from Cy5.5 in organs and brain was detected by IVIS. Comparisons of the delivery efficiency were against the USuntreated using Student's $t$-test $(n=2) . * P<0.05$ and $* * P<0.005$.

Abbreviations: IVIS, in vivo imaging system; NIR-Alb NP, near-infrared dye-incorporated albumin nanoparticle; US, ultrasound.

luc cells significantly increased luciferase activity. To create the U87MG orthotopic xenograft mouse model, $2 \times 10^{5}$ U87MG-luc cells were intracranially injected into mice, and the tumor cells were allowed to grow for 3 days after the implantation. We monitored luminescence from the implanted U87MG-Luc cells by IVIS. Then, in 4 of 6 mice, the cells were placed and grown in the brain. US was applied to the orthotopic mice under the optimized US parameters $\left(0.1 \mathrm{~W} / \mathrm{cm}^{2}, 10 \%\right.$ duty cycle, and 5-min treatment) immediately after tail vein injection of NIR-Alb NP complex solution (Scheme 1B). Twenty-four hours after the treatment, the mice were sacrificed. The five major organs and brain were collected and evaluated by IVIS. In Figure 6, NIR-Alb NPs were mainly detected in the liver and were also detected in the lung, kidney, spleen, and brain but not the heart. In brains, the intensity of the US-treated groups was 1.5 times higher than the US-nontreated group, with statistical significance being observed. Brain damages, such as hemorrhage and swelling, were not observed.

\section{Discussion}

Fluorescent analysis is a good tool for biology and medicine because of the capabilities of qualitative and quantitative analyses. Biological and medical samples, such as tissues and organs, have intrinsic fluorescence, known as autofluorescence. This autofluorescence is a critical issue for fluorescent imaging. One way to enhance the signal is to use NIR fluorescence, which can reduce photon scattering, light absorption, and autofluorescence; in addition, it affords high imaging resolution with increasing tissue penetration depths. ${ }^{28}$ Considering the enhancement of the signal, creation of chemical bonding, and instrumental compatibility, Cy5.5 dye derivatives were the best in our system. Also, the commercially available, reactive NHSfunctionalized Cy5.5 dye was quickly coupled with amine groups of the albumin NP without physicochemical changes of the dye.

We synthesized albumin NPs using human serum albumin by the well-known desolvation method. ${ }^{26}$ Highly reactive glutaraldehyde was used as a cross-linking agent to make chemical bonding between amines in albumins. Potential toxicity by glutaraldehyde was eliminated by a multiple purification process. As shown in cell viability tests, our NIR-Alb NPs did not show any acute toxicity in the four cell lines. Prakash et al demonstrated that $143 \mathrm{~nm}$ sized HSA NPs showed $85.4 \%$ of cell viability in a MCF-7 cell line for $48 \mathrm{hrs} .{ }^{29}$ Wei et al tested $195 \mathrm{~nm}$-sized BSA NPs to $300 \mu \mathrm{g} / \mathrm{mL}$ concentration in for $36 \mathrm{hrs}$ in a MBR 262-1 cell line, and the BSA NP was more than $90 \%$ in cell viability. ${ }^{30}$ In line with other groups' results, albumin NPs were strongly biocompatible materials, regardless of the origin of albumin, human, or bovine.

Complexation of NIR-Alb NP and MB used amide bonding by the well-known coupling reaction between the amine group in NIR-Alb NPs and NHS-activated carboxylic acid in MBs. To perform the complexation, the MB was first activated by Vialmix, and then NIR-Alb NPs was added. The mixing ratio and complexation time were optimized by quantification of the un-complexed NIR-Alb NPs. DLS was not a well-characterized tool for the complexation 
because the size change between MBs and the complexes varied in size distribution. TEM was one of the great characterization tools for the complex. However, our MB composed of two types of phospholipids could not be observed by TEM. After staining, both MBs and complexes could be successfully observed (Figure 1). In our expected structure of the NIR-Alb NP complex in Scheme 1, it was clearly confirmed that the NIR-Alb NPs were surrounded on the $\mathrm{MB}$, and after the complexation, its size increase was confirmed by DLS analysis.

In our study, the most important factor for the BBB opening was intensity. Lower intensity was better due to the MB cavitation. ${ }^{19}$ We did not observe a significant change by varying duty cycle at the $0.1 \mathrm{~W} / \mathrm{cm}^{2}$ intensity (Figure 5). Skin burning and brain swelling were observed under the higher intensity with $100 \%$ duty cycle, while no damage was observed at the low intensity with $100 \%$ duty cycle (Figure S5). Our unfocused US affected the whole brain, leading to swelling. At the highest intensity, $5 \mathrm{~W} /$ $\mathrm{cm}^{2}$, serious hemorrhage in the brain parenchyma was observed. Interestingly, even though exposed to the same US, mice without the MB injection did not show any brain damage or death. The mice were healthy. Overall, we demonstrated that unfocused US could disrupt the BBB with MB injection.

\section{Conclusion}

For the BBB opening and NP delivery at the same time, we synthesized US-sensitizing NP complexes via chemical binding between MBs and NPs and NIR-Alb NPs for fluorescent detection. The NIR-Alb NP complex, NPsdecorated MB was directly confirmed by TEM. The human-derived, biocompatible NIR-Alb NPs were tested to a $500 \mu \mathrm{g} / \mathrm{mL}$ dose for 3 days in four brain cell lines and did not show any significant toxicity. In the in vivo animal study, the efficient US parameters $\left(0.1 \mathrm{~W} / \mathrm{cm}^{2}\right.$, $10 \%$ duty cycle, and 5-min treatment) were found without any brain damage, such as hemorrhage and swelling. To evaluate delivery efficiency, we created and confirmed a U87MG orthotopic mouse model by luminescence monitoring. We showed that the fluorescence intensity in the brain was 1.5 times higher than in the US-untreated group when the optimized US parameters were applied. Overall, we demonstrated that our system, US-sensitizing NP complex (NIR-Alb NP complex) and US, could open the BBB and effectively deliver the NIR-Alb NP to the brain. This delivery system by US may be a great therapeutic method to overcome the limitation of drug delivery to the brain.

\section{Acknowledgments}

This research was supported by a grant (D171751) from Gyeonggi Technology Development Program funded by Gyeonggi Province and by grant no 13-2017-003 from the SNUBH Research Fund to CYK.

\section{Disclosure}

The authors report no conflicts of interest in this work.

\section{References}

1. Ostrom QT, Gittleman H, Liao P, et al. CBTRUS statistical report: primary brain and other central nervous system tumors diagnosed in the United States in 2010-2014. Neuro Oncol. 2017;19(suppl_5):v1-v88. doi 10.1093/neuonc/nox 158

2. Pardridge WM. The blood-brain barrier: bottleneck in brain drug development. NeuroRx. 2005;2(1):3-14. doi:10.1602/neurorx.2.1.3

3. Bulbake U, Doppalapudi S, Kommineni N, Khan W. Liposomal formulations in clinical use: an updated review. Pharmaceutics. 2017;9:2. doi:10.3390/pharmaceutics9020012

4. Shafei A, El-Bakly W, Sobhy A, et al. A review on the efficacy and toxicity of different doxorubicin nanoparticles for targeted therapy in metastatic breast cancer. Biomed Pharmacother. 2017;95:1209-1218. doi:10.1016/j.biopha.2017.09.059

5. Nakamura Y, Mochida A, Choyke PL, Kobayashi H. Nanodrug delivery: is the enhanced permeability and retention effect sufficient for curing cancer? Bioconjug Chem. 2016;27(10):2225-2238. doi:10.1021/acs.bioconjchem.6b00417

6. Chen Y, Liu L. Modern methods for delivery of drugs across the blood-brain barrier. Adv Drug Deliv Rev. 2012;64(7):640-665. doi:10.1016/j.addr.2011.11.010

7. Rowe RC, Sheskey PJ, Cook WG, Fenton ME. Handbook of Pharmaceutical Excipients. 7th ed. London: Pharmaceutical Press; 2012.

8. Rempel SA, Golembieski WA, Fisher JL, Maile M, Nakeff A. SPARC modulates cell growth, attachment and migration of U87 glioma cells on brain extracellular matrix proteins. J Neurooncol. 2001;53(2):149-160. doi:10.1023/A:1012201300188

9. Golembieski WA, Ge S, Nelson K, Mikkelsen T, Rempel SA. Increased SPARC expression promotes U87 glioblastoma invasion in vitro. Int J Dev Neurosci. 1999;17(5-6):463-472. doi:10.1016/ S0736-5748(99)00009-X

10. Tsou YH, Zhang XQ, Zhu H, Syed S, Xu X. Drug delivery to the brain across the blood-brain barrier using nanomaterials. Small. 2017;13:43. doi:10.1002/smll.201701921

11. Quadri SA, Waqas M, Khan I, et al. High-intensity focused ultrasound: past, present, and future in neurosurgery. Neurosurg Focus. 2018;44(2):E16. doi:10.3171/2017.11.FOCUS17610

12. Lipsman N, Meng Y, Bethune AJ, et al. Blood-brain barrier opening in Alzheimer's disease using MR-guided focused ultrasound. Nat Commun. 2018;9(1):2336. doi:10.1038/s41467-018-04529-6

13. Choi JJ, Pernot M, Small SA, Konofagou EE. Noninvasive, transcranial and localized opening of the blood-brain barrier using focused ultrasound in mice. Ultrasound Med Biol. 2007;33(1):95-104. doi:10.1016/j.ultrasmedbio.2006.07.018

14. McDannold N, Arvanitis CD, Vykhodtseva N, Livingstone MS. Temporary disruption of the blood-brain barrier by use of ultrasound and microbubbles: safety and efficacy evaluation in rhesus macaques. Cancer Res. 2012;72(14):3652-3663. doi:10.1158/0008-5472.CAN-12-0128 
15. Sun T, Zhang Y, Power C, et al. Closed-loop control of targeted ultrasound drug delivery across the blood-brain/tumor barriers in a rat glioma model. Proc Natl Acad Sci U S A. 2017;114(48): E10281-E10290. doi:10.1073/pnas.1713328114

16. Wu SK, Chu PC, Chai WY, et al. Characterization of different microbubbles in assisting focused ultrasound-induced blood-brain barrier opening. Sci Rep. 2017;7:46689. doi:10.1038/srep46689

17. Sun T, Sutton JT, Power C, Zhang Y, Miller EL, McDannold NJ. Transcranial cavitation-mediated ultrasound therapy at sub-MHz frequency via temporal interference modulation. Appl Phys Lett. 2017;111(16):163701. doi:10.1063/1.5000896

18. Hynynen K, McDannold N, Sheikov NA, Jolesz FA, Vykhodtseva N. Local and reversible blood-brain barrier disruption by noninvasive focused ultrasound at frequencies suitable for trans-skull sonications. Neuroimage. 2005;24(1):12-20. doi:10.1016/j.neuroimage.2004. 06.046

19. Hynynen K, McDannold N, Vykhodtseva N, Jolesz FA. Noninvasive MR imaging-guided focal opening of the blood-brain barrier in rabbits. Radiology. 2001;220(3):640-646. doi:10.1148/radiol.2202001804

20. Song KH, Fan AC, Hinkle JJ, Newman J, Borden MA, Harvey BK. Microbubble gas volume: a unifying dose parameter in blood-brain barrier opening by focused ultrasound. Theranostics. 2017;7 (1):144-152. doi:10.7150/thno.15987

21. Jones RM, Deng L, Leung K, McMahon D, O’Reilly MA, Hynynen K. Three-dimensional transcranial microbubble imaging for guiding volumetric ultrasound-mediated blood-brain barrier opening. Theranostics. 2018;8(11):2909-2926. doi:10.7150/thno.24911

22. Shin J, Kong C, Cho JS, et al. Focused ultrasound-mediated noninvasive blood-brain barrier modulation: preclinical examination of efficacy and safety in various sonication parameters. Neurosurg Focus. 2018;44(2):E15. doi:10.3171/2017.11. FOCUS17627
23. Zhao B, Chen Y, Liu J, et al. Blood-brain barrier disruption induced by diagnostic ultrasound combined with microbubbles in mice. Oncotarget. 2018;9(4):4897-4914. doi:10.18632/ oncotarget. 23527

24. Yao L, Song Q, Bai W, et al. Facilitated brain delivery of poly (ethylene glycol)-poly (lactic acid) nanoparticles by microbubble-enhanced unfocused ultrasound. Biomaterials. 2014;35(10):3384-3395. doi:10.1016/j.biomaterials.2014.0 1.026

25. Bing KF, Howles GP, Qi Y, Palmeri ML, Nightingale KR. Bloodbrain barrier (BBB) disruption using a diagnostic ultrasound scanner and definity in mice. Ultrasound Med Biol. 2009;35 (8):1298-1308. doi:10.1016/j.ultrasmedbio.2009.03.012

26. Langer K, Balthasar S, Vogel V, Dinauer N, von Briesen $H$, Schubert D. Optimization of the preparation process for human serum albumin (HSA) nanoparticles. Int J Pharm. 2003;257(1-2):169-180. doi:10.1016/S0378-5173(03)00134-0

27. Tinkov S, Coester C, Serba S, et al. New doxorubicin-loaded phospholipid microbubbles for targeted tumor therapy: in-vivo characterization. J Control Release. 2010;148(3):368-372. doi:10. 1016/j.jconrel.2010.09.004

28. Hong G, Antaris AL, Dai H. Near-infrared fluorophores for biomedical imaging. Nat Biomed Eng. 2017;1:0010. doi:10.1038/s41551016-0010

29. Lomis N, Westfall S, Farahdel L, Malhotra M, Shum-Tim D, Prakash S. Human serum albumin nanoparticles for use in cancer drug delivery: process optimization and in vitro characterization. Nanomater. 2016;6:6. doi:10.3390/nano6060116

30. Wei KC, Lin FW, Huang CY, et al. 1,3-Bis(2-chloroethyl)-1-nitrosourea-loaded bovine serum albumin nanoparticles with dual magnetic resonance-fluorescence imaging for tracking of chemotherapeutic agents. Int J Nanomedicine. 2016;11:4065-4075. doi:10.2147/IJN.S113589

\section{Publish your work in this journal}

The International Journal of Nanomedicine is an international, peerreviewed journal focusing on the application of nanotechnology in diagnostics, therapeutics, and drug delivery systems throughout the biomedical field. This journal is indexed on PubMed Central, MedLine, CAS, SciSearch ${ }^{\mathbb{B}}$, Current Contents ${ }^{\circledR} /$ Clinical Medicine,
Journal Citation Reports/Science Edition, EMBase, Scopus and the Elsevier Bibliographic databases. The manuscript management system is completely online and includes a very quick and fair peer-review system, which is all easy to use. Visit http://www.dovepress.com/ testimonials.php to read real quotes from published authors. 